\title{
Effects of nonsphericity on the behavior of Lorenz-Mie resonances in scattering characteristics of liquid-cloud droplets
}

\author{
Janna M. Dlugach a , Michael I. Mishchenko ${ }^{\mathrm{b}, *}$ \\ ${ }^{a}$ Main Astronomical Observatory of the National Academy of Sciences of Ukraine, 27 Zabolotny Street, 03680 Kyiv, Ukraine \\ ${ }^{\mathrm{b}}$ NASA Goddard Institute for Space Studies, 2880 Broadway, New York, NY 10025, USA
}

\section{A R T I C L E I N F O}

\section{Article history:}

Received 6 November 2013

Received in revised form

31 December 2013

Accepted 7 January 2014

Available online 15 January 2014

Keywords:

Lorenz-Mie resonances

Averaging over sizes

Averaging over orientations

Scattering characteristics

Nonspherical particles

Cloud droplets

T-matrix method

\begin{abstract}
A B S T R A C T
By using the results of highly accurate $T$-matrix computations for randomly oriented oblate and prolate spheroids and Chebyshev particles with varying degrees of asphericity, we analyze the effects of a deviation of water-droplet shapes from that of a perfect sphere on the behavior of Lorenz-Mie morphology-dependent resonances of various widths. We demonstrate that the positions and profiles of the resonances can change significantly with increasing asphericity. The absolute degree of asphericity required to suppress a Lorenz-Mie resonance is approximately proportional to the resonance width. Our results imply that numerical averaging of scattering characteristics of real cloud droplets over sizes may rely on a significantly coarser size-parameter resolution than that required for ideal, perfectly spherical particles.
\end{abstract}

Published by Elsevier Ltd.

\section{Introduction}

Liquid-cloud droplets always exist in the atmosphere in the form of polydisperse mixtures. Therefore, the numerical modeling of their scattering and absorption properties (such as the optical cross sections, single-scattering albedo, and scattering matrix elements) must involve averaging over a size distribution by using an appropriate quadrature integration formula. For a given type of quadrature formula, the number of the quadrature division points and the total integration range determine the respective size-parameter resolution $\Delta x$, where $x=2 \pi r / \lambda$, $r$ is the sphere radius, and $\lambda$ is the wavelength in the surrounding medium. In principle, the size-parameter resolution should be made finer (i.e., $\Delta x$ should be

\footnotetext{
* Corresponding author. Fax: +1212678 5622

E-mail address: crmim2@gmail.com (M.I. Mishchenko).
}

decreased) iteratively until the succession of the corresponding numerical results converges to a certain constant value. However, the straightforward practical implementation of this procedure may require a substantial or even prohibitive computer time. Therefore, the size parameter resolution is usually selected based on the efficiency of the available computers rather than on an objective accuracy criterion. At the same time, it has been claimed that the size-averaged results obtained at canonical size-parameter resolutions cannot be trusted (see, e.g., Ref. [1]). The main reason for requiring an exceedingly small $\Delta x$ in Lorenz-Mie computations for perfect spheres is the existence of numerous morphology-dependent resonances (MDRs) [2-4], some of which are super-narrow features that can be extremely difficult to resolve numerically.

The results of an extensive analysis of potential effects of size-parameter resolution on the outcome of polydisperse Lorenz-Mie computations for liquid-water clouds were published in Ref. [5]. However, the final conclusions 
were based on the assumption of a perfect spherical shape of the cloud droplets and on the ignorance of the fact that the actual droplet shapes can be expected to deviate from that of a perfect sphere due to various ambient disturbances typical of a naturally-occurring cloud. On the other hand, it was shown in Ref. [6] that for spheroids and Chebyshev particles with a refractive index of $m=1.4$, the deformation of a sphere by as little as one hundredth of a wavelength can essentially annihilate super-narrow MDRs. Obviously, this factor can be very important in selecting an appropriate value of $\Delta x$ when performing polydisperse computations for actual liquid-cloud droplets.

It is well known that the shape of falling raindrops with radii exceeding $\sim 1 \mathrm{~mm}$ can be quite nonspherical [7]. On the other hand, experimental data on the likely degree of asphericity of typical liquid-cloud droplets with radii ranging from a few to $\sim 20 \mu \mathrm{m}$ appear to be absent. This shortage of critical information makes impossible a definitive solution of the problem of selecting an appropriately fine size-parameter
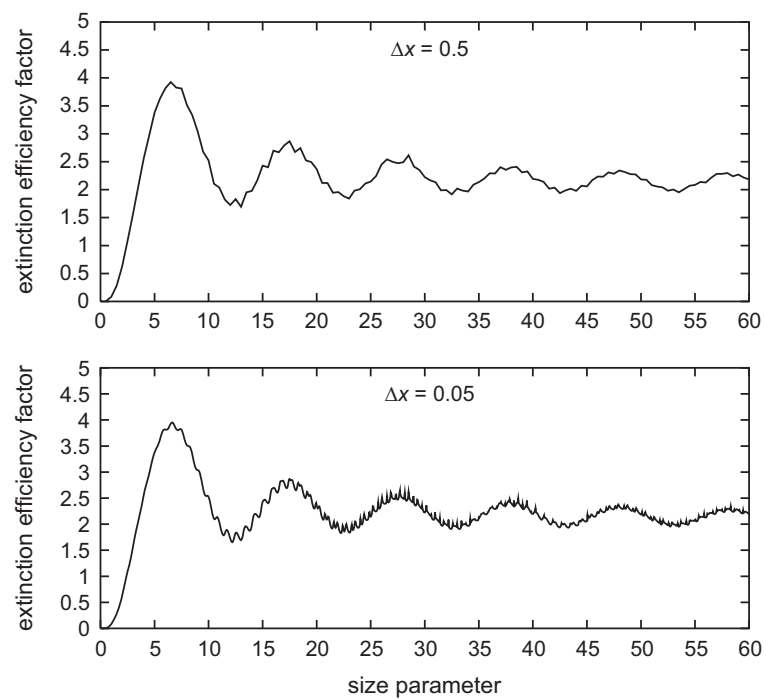

Fig. 1. Extinction efficiency factor $Q_{\text {ext }}$ versus size parameter $x$ for a perfectly spherical particle with the refractive index $m=1.31$. The results are plotted using size parameter resolutions $\Delta x=0.5$ (top panel) and 0.05 (lower panel).

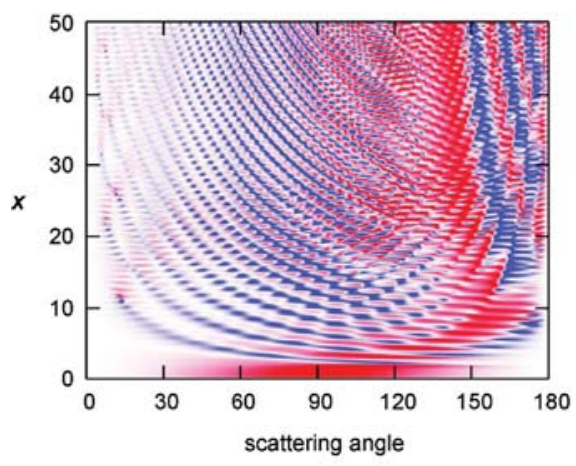

resolution $\Delta x$. However, we can facilitate the eventual solution of this problem by performing a systematic analysis of the likely effects of increasing deviations of the shapes of liquid-cloud droplets from perfect sphericity on the behavior of Lorenz-Mie MDRs of varying widths. Such an analysis is the main objective of this note.

\section{Preliminaries}

All numerical results reported and discussed below are based on using a fixed relative refractive index of $m=1.31$. This value was selected to represent the real part of the refractive index of liquid water in a wide spectral range extending from near ultraviolet to short-wave infrared wavelengths [8]. For simplicity, the imaginary part is always taken to be zero. It is well known that even small amounts of absorption serve to effectively suppress MDRs [4]. Thus by ignoring absorption we, in essence, consider the worst case scenario in terms of the potential effect of MDRs on the result of numerical integration over cloud-droplet radii using course size-parameter resolution.

To illustrate the potential effects of a course size-parameter resolution, Fig. 1 depicts the dimensionless extinction efficiency factor $Q_{\text {ext }}=C_{\text {ext }} /\left(\pi r^{2}\right)$ (where $C_{\text {ext }}$ is the scattering cross section [4]) versus size parameter $x$ for a perfectly spherical particle plotted using two values of the size parameter step size: $\Delta x=0.5$ and 0.05 . It is seen indeed that the bottom curve reveals quite vividly numerous spikelike MDRs superimposed on the nearly periodic interference structure. The latter is traditionally explained as the result of interference of light diffracted and transmitted by the particle $[9,10]$. On the other hand, the resonances are attributed to the fact that when the size parameter $x$ approaches a resonant value $x_{\text {res }}$, the denominator in the formula for a Mie coefficient $a_{n}$ or $b_{n}$ approaches a local minimum, thereby causing the appearance of a local extremum in the curve for a specific scattering characteristic (see., e.g., Ref. [3] and references therein).

Fig. 2 is another way to look at the optical resonances. It depicts the degree of linear polarization of scattered light for unpolarized incident light, $-F_{21} / F_{11}$ (where $F_{11}$ and $F_{21}$ are elements of the normalized Stokes scattering matrix [4]), as a function of the scattering angle $\Theta$ and size parameter $x$.

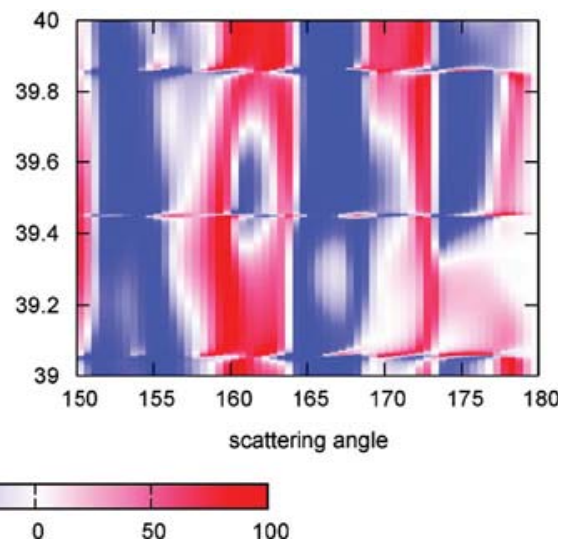

Fig. 2. Low-resolution (left) and medium-resolution (right) images of the degree of linear polarization $-F_{21} / F_{11}(\%)$ as a function of size parameter $x$ and scattering angle $\Theta(\mathrm{deg})$ for a perfectly spherical particle with the refractive index $m=1.31$. 
The left-hand panel is plotted with low resolution and is obviously dominated by the interference structure. The right-hand panel is a zoomed, medium-resolution image of

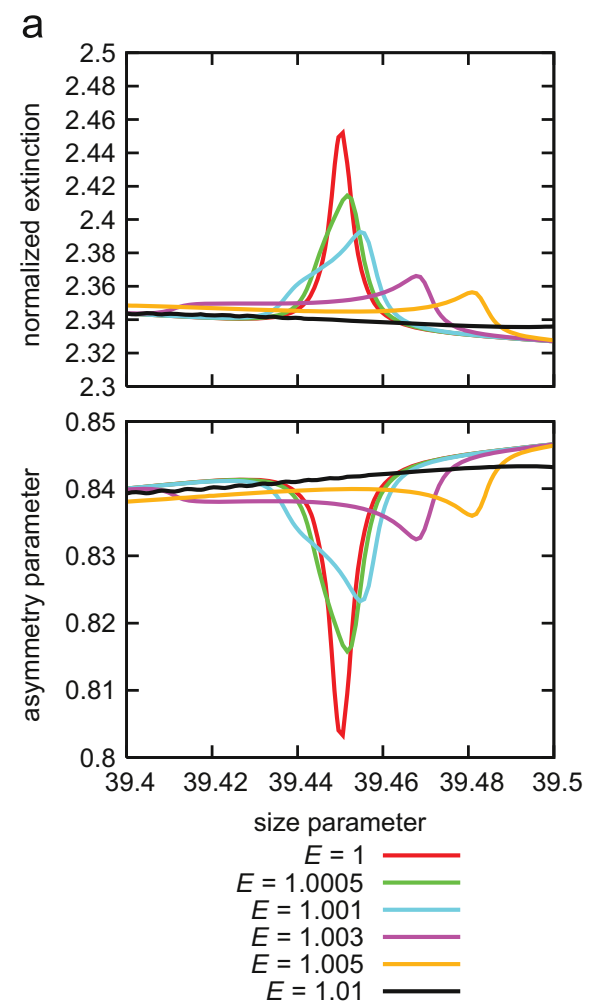

b
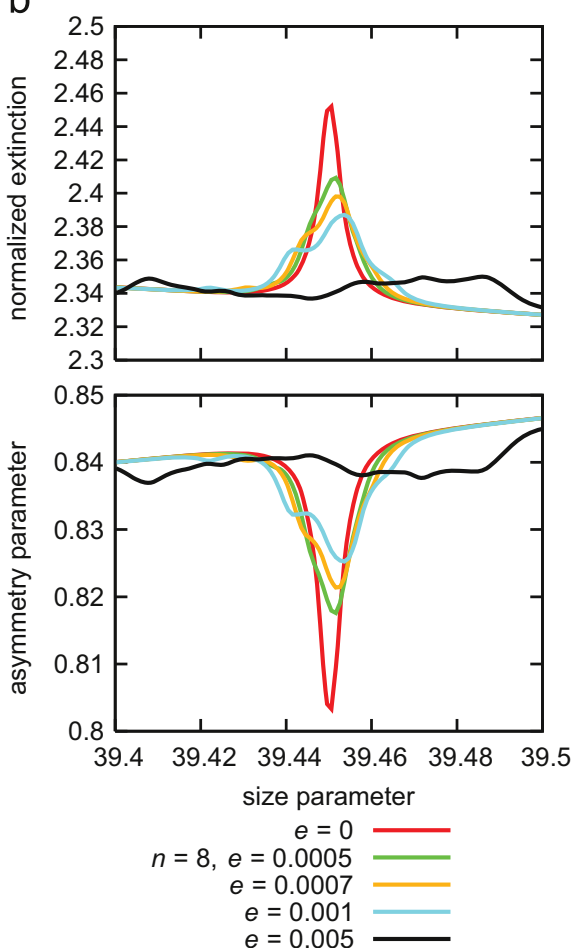

a small part of the left-hand panel and reveals fine ripple features and sharp dislocations; the latter are first indicators of super-narrow MDRs.
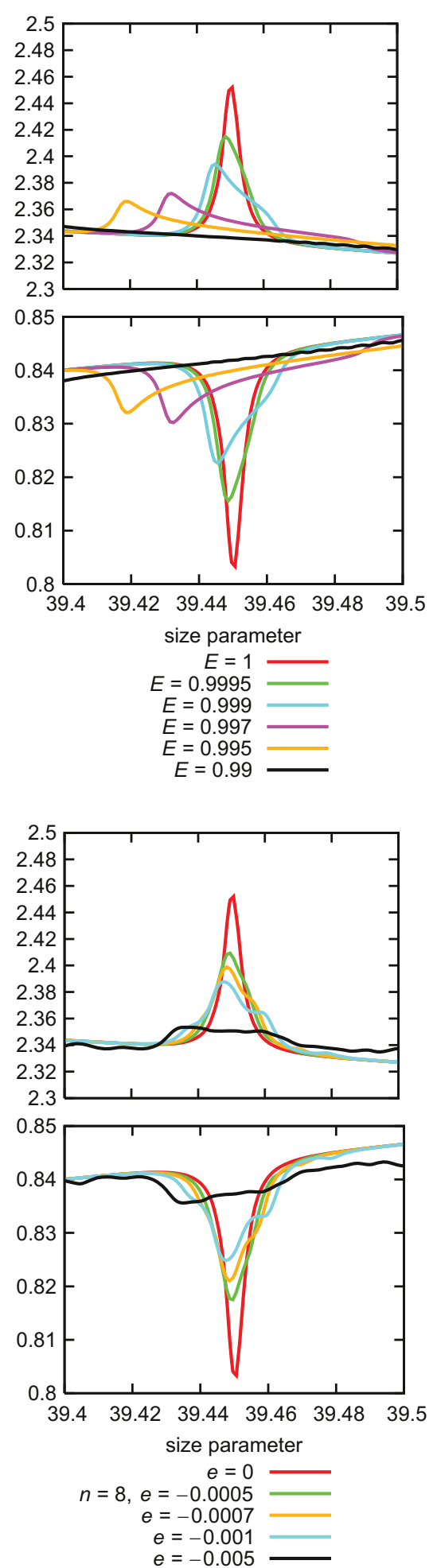

Fig. 3. (a) Normalized extinction and asymmetry parameter versus size parameter in the vicinity of the $x_{\text {res }}=39.4502$ resonance for a perfect sphere and volume-equivalent, randomly oriented oblate (left-hand column) and prolate (right-hand column) spheroids. (b) The same, but for volume-equivalent, randomly oriented Chebyshev particles with positive (left-hand column) and negative (right-hand column) values of the deformation parameter. 
In order to investigate the effects of deviations of the droplet shape from that of a perfect sphere on the behavior of the Lorenz-Mie MDRs, it is necessary to perform extremely accurate computations of scattering

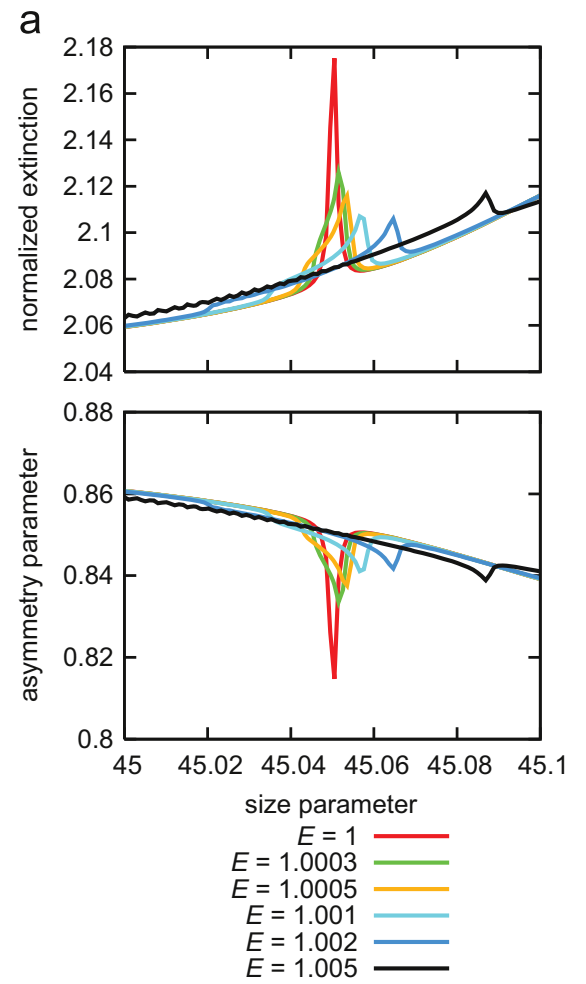

b
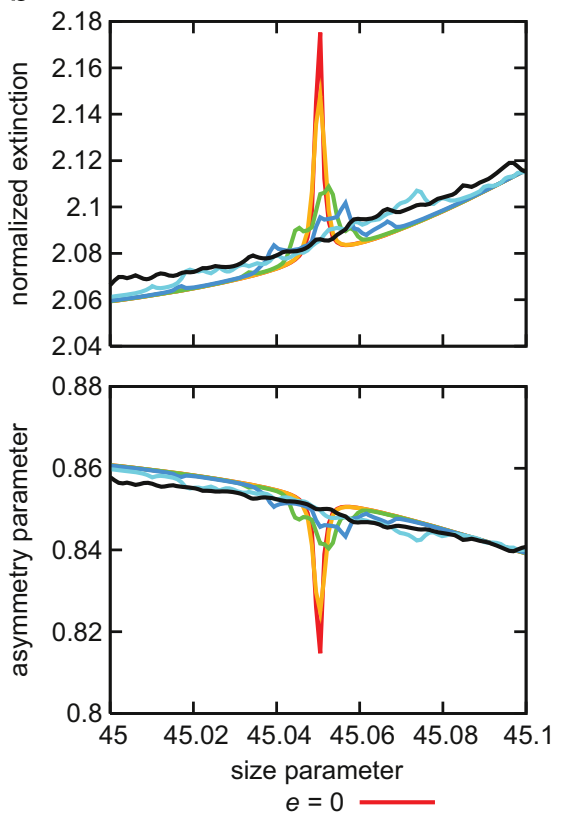

$n=8, e=0.0001$

$e=0.0005$

$e=0.001$

$e=0.003$

$e=0.005$ properties of randomly oriented nonspherical particles over a substantial range of size parameters. In this study, such computations are performed by using the extendedprecision version of the FORTRAN T-matrix program [11]
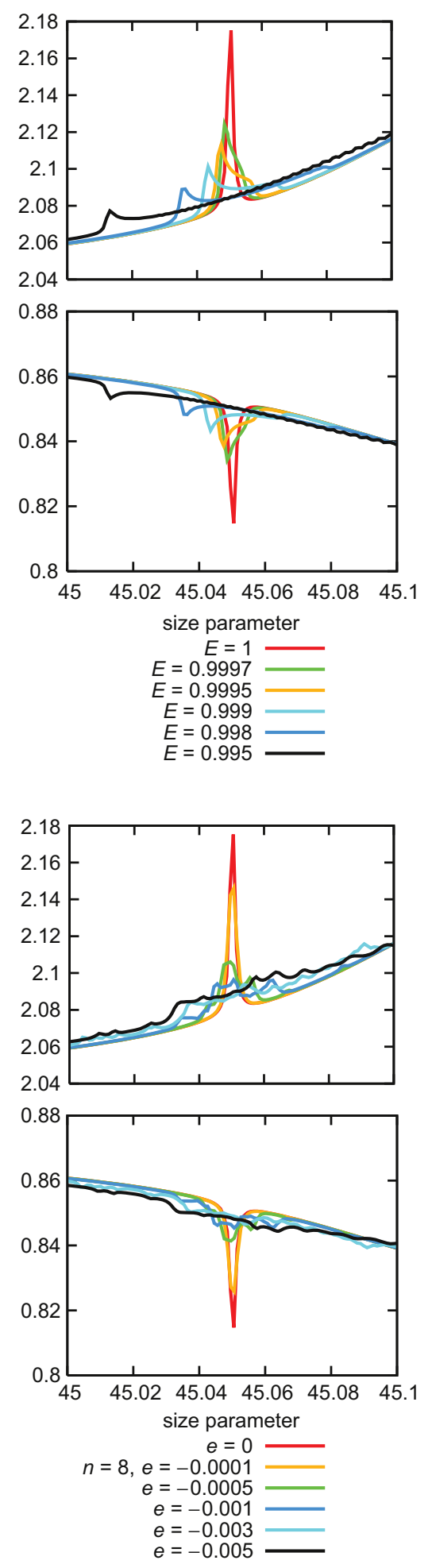

Fig. 4. As in Fig. 3, but in the vicinity of the $x_{\text {res }}=45.0502$ resonance. 
based on Waterman's extended boundary condition method [12]. In order to model particle aspherisity, we use oblate and prolate spheroids and high-order Chebyshev particles [13]. Prolate and oblate spheroidal shapes are intended to represent uniform deformations of a spherical droplet caused by stretching or flattening of the droplet as a whole, whereas the shape of a Chebyshev particle is used to simulate microscopic undulations on the droplet surface. The shape of a spheroid is characterized by the axis ratio $E=a / b$, where $b$ is the spheroid semi-axis along the axis of rotation and $a$ is the semi-axis in the perpendicular direction. The shape of a Chebyshev particle with respect to the particle reference frame with the $z$-axis serving as the axis of rotational symmetry is given by $r(\theta)=$ $r_{0}\left[1+e T_{n}(\cos \theta)\right]$, where $r_{0}$ is the radius of the unperturbed sphere, $e$ is the relative deformation parameter, $T_{n}(\cos \theta)=\cos n \theta$ is the Chebyshev polynomial of degree $n$, and $\theta$ is the polar angle.

\section{Numerical modeling and discussion}

Figs. 3-5 show the results of $T$-matrix computations of the normalized extinction and asymmetry parameter for spheres, randomly oriented volume-equivalent spheroids, and volume-equivalent Chebyshev particles in the range of equivalent-volume-sphere size parameters affected by three Lorenz-Mie resonances, viz., $b_{46}^{1}\left(x_{\text {res }}=39.4502\right.$, $\left.W_{x}=0.003\right) ; \quad b_{53}^{1} \quad\left(x_{\mathrm{res}}=45.0502, W_{x}=0.0009\right) ;$ and $a_{63}^{1}$ $\left(x_{\text {res }}=53.4408, W_{x}=0.0002\right)$. Here, $W_{x}$ is the half-width at half maximum of a Lorenz-Mie resonance in units of size parameter, while the notation $b_{46}^{1}, b_{53}^{1}$, and $a_{63}^{1}$ is intended to label the first resonances caused by the $b_{46}$, $b_{53}$, and $a_{63}$ Lorenz-Mie coefficients as $x$ increases from zero [14]. The normalized extinction is defined as the ratio $\left\langle C_{\text {ext }}\right\rangle /\left(\pi r^{2}\right)$, where $\left\langle C_{\text {ext }}\right\rangle$ is the orientation-averaged extinction cross section and $r$ is the radius of the equalvolume sphere. For spherical particles this ratio coincides with the extinction efficiency factor. The asymmetry parameter is defined by

$\langle\cos \Theta\rangle=\frac{1}{2} \int_{0}^{\pi} d \Theta \sin \Theta F_{11}(\Theta) \cos \Theta$.

The relative accuracy of the $T$-matrix computations was set to be better than $10^{-9}$. The values of resonance locations and half-widths were calculated with the Fortran computer program developed by S. Hill (personal communication) using the algorithm described in Ref. [15]. It is seen that the half-width values of these resonances progressively decrease.

Fig. 3(a) demonstrates the resulting behavior of the normalized extinction and asymmetry parameter in the vicinity of the $x_{\text {res }}=39.4502$ resonance for spheres and oblate and prolate spheroids with different values of the axis ratio $E$. In Fig. 3(b), the corresponding size-parameter dependences are depicted for Chebyshev particles with $n=8$ and various positive and negative values of the relative deformation parameter $e$. The reader can see that all types of droplet deformations have a similar suppressing effect on the LorenzMie resonance considered. Specifically, for spheroids increasing the degree of asphericity results in a rapid decrease of the resonance peak in the normalized extinction and the depth of the spike-like minimum in the asymmetry parameter. Additionally, an increase of asphericity results in shifting the location of the resonance to either larger (oblate spheroids) or smaller (prolate spheroids) values of the volume-equivalentsphere size parameter. Interestingly, the curves for oblate and prolate spheroids corresponding to the same degree of asphericity look almost like mirror images of each other. A similar suppressing effect of increasing asphericity of Ghebyshev particles with positive and negative values of the deformation parameter is clearly seen in Fig. 3(b). However, unlike the case for spheroids, increasing the absolute value of the deformation parameter $e$ results in a growing complexity of the fine resonance structure, whereas the position of the main resonance appears to be much more stable. The curves for $|e|=0.005$ show that the corresponding absolute degree of asphericity not only annihilates the main resonance but starts to affect the background values of the normalized extinction and asymmetry parameter.

Figs. 4 and 5 reveal qualitatively similar effects of growing asphericity on MDRs with decreasing half-widths. More importantly, however, it demonstrates that it takes smaller absolute degrees of asphericity to "kill" narrower MDRs.

Figs. 3-5 show that a resonance peak in extinction is always accompanied by a resonance spike-like minimum in the asymmetry parameter. This trait can easily be explained by taking into account that, according to Refs. $[16,17]$, the Lorenz-Mie coefficients can be expressed as

$$
a_{n}(x, m)=\frac{\left[p_{n}(x, m)\right]^{2}}{\left[p_{n}(x, m)\right]^{2}+\left[q_{n}(x, m)\right]^{2}}-i \frac{p_{n}(x, m) q_{n}(x, m)}{\left[p_{n}(x, m)\right]^{2}+\left[q_{n}(x, m)\right]^{2}},
$$

$b_{n}(x, m)=\frac{\left[r_{n}(x, m)\right]^{2}}{\left[r_{n}(x, m)\right]^{2}+\left[s_{n}(x, m)\right]^{2}}-i \frac{r_{n}(x, m) s_{n}(x, m)}{\left[r_{n}(x, m)\right]^{2}+\left[s_{n}(x, m)\right]^{2}}$.

The functions $p_{n}(x, m), q_{n}(x, m), \quad r_{n}(x, m)$, and $s_{n}(x, m)$ are given by Eqs. (9.3)-(9.6) of Ref. [4] and are real if the relative refractive index $m$ is real. What happens at a resonance is that either $q_{n}(x, m)$ or $s_{n}(x, m)$ vanishes. For this specific set of $x-, m-$, and $n$-values, the real part of $a_{n}(x, m)$ or $b_{n}(x, m)$ reaches its maximum possible value, unity, while the imaginary part vanishes [2]. Let us now recall that

$\langle\cos \Theta\rangle=\frac{4 \pi}{k_{1}^{2} C_{\text {sca }}} \operatorname{Re} \sum_{n=1}^{\infty}\left[\frac{n(n+2)}{n+1}\left(a_{n} a_{n+1}^{*}+b_{n} b_{n+1}^{*}\right)+\frac{2 n+1}{n(n+1)} a_{n} b_{n}^{*}\right]$

and, for nonabsorbing particles,

$C_{\mathrm{ext}}=C_{\mathrm{sca}}=\frac{2 \pi}{k_{1}^{2}} \sum_{n=1}^{\infty}(2 n+1)\left\{\left|a_{n}\right|^{2}+\left|b_{n}\right|^{2}\right\}$.

If, for example, the Lorenz-Mie coefficient $a_{n}$ with $n \gg 1$ has a resonance at the size parameter $x_{\text {res }}$ [i.e., $a_{n}\left(x_{\text {res }}, m\right)=1$ ] then, obviously, it causes a peak centered at $x_{\text {res }}$ in the plot of $C_{\text {ext }}$ (as well as that of $C_{\text {sca }}$ ) as a function of size parameter. Furthermore, one can expect that at the resonance size parameter,

$\operatorname{Re}\left[a_{n+1}^{*}+b_{n} b_{n+1}^{*}+\frac{2}{n^{2}} b_{n}^{*}\right] \ll 1+\left|b_{n}\right|^{2}$. 

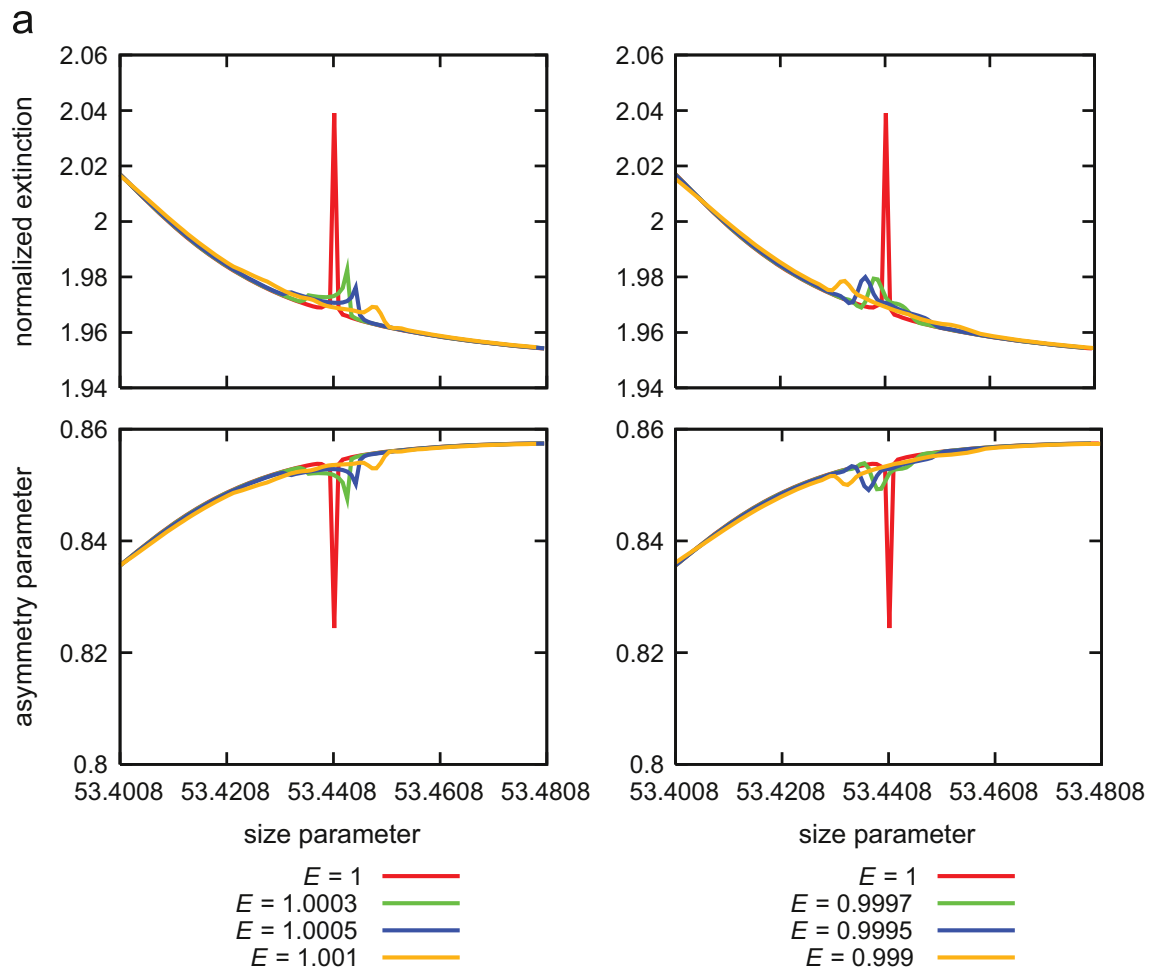

b
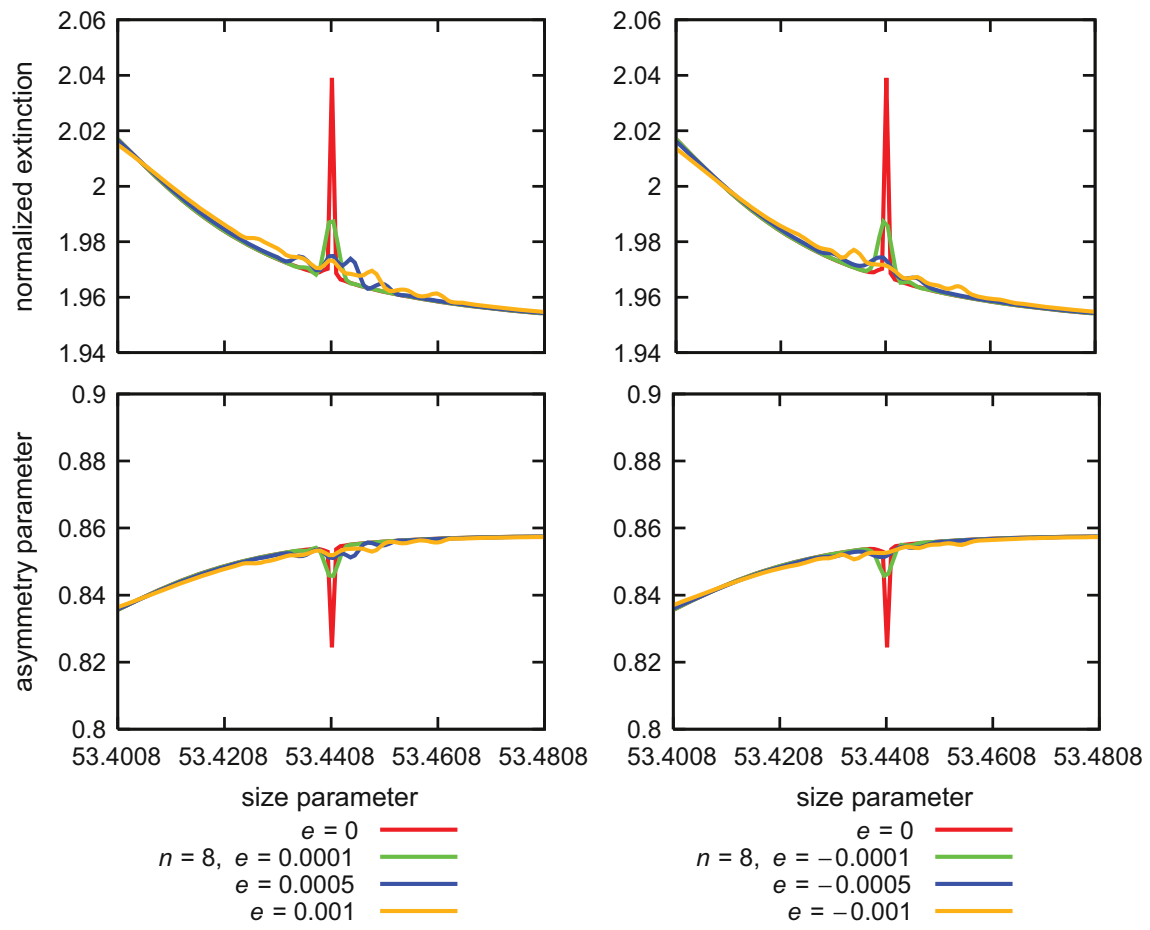

Fig. 5. As in Fig. 3, but in the vicinity of the $x_{\text {res }}=53.4408$ resonance.

This implies that the peak in $C_{\text {sca }}$ will cause a resonance minimum in $\langle\cos \Theta\rangle$. A corollary of this explanation is that there will be a resonance peak in the plot of the radiationpressure cross section $C_{\mathrm{pr}}=C_{\mathrm{ext}}(1-\langle\cos \Theta\rangle)$.
Figs. 6 and 7 depict the results of computations of the degree of linear polarization in the vicinity of the $x_{\text {res }}=39.4502$ MDR for scattering angles in the range $165^{\circ} \leq \Theta \leq 175^{\circ}$. It is seen again that increasing asphericity 

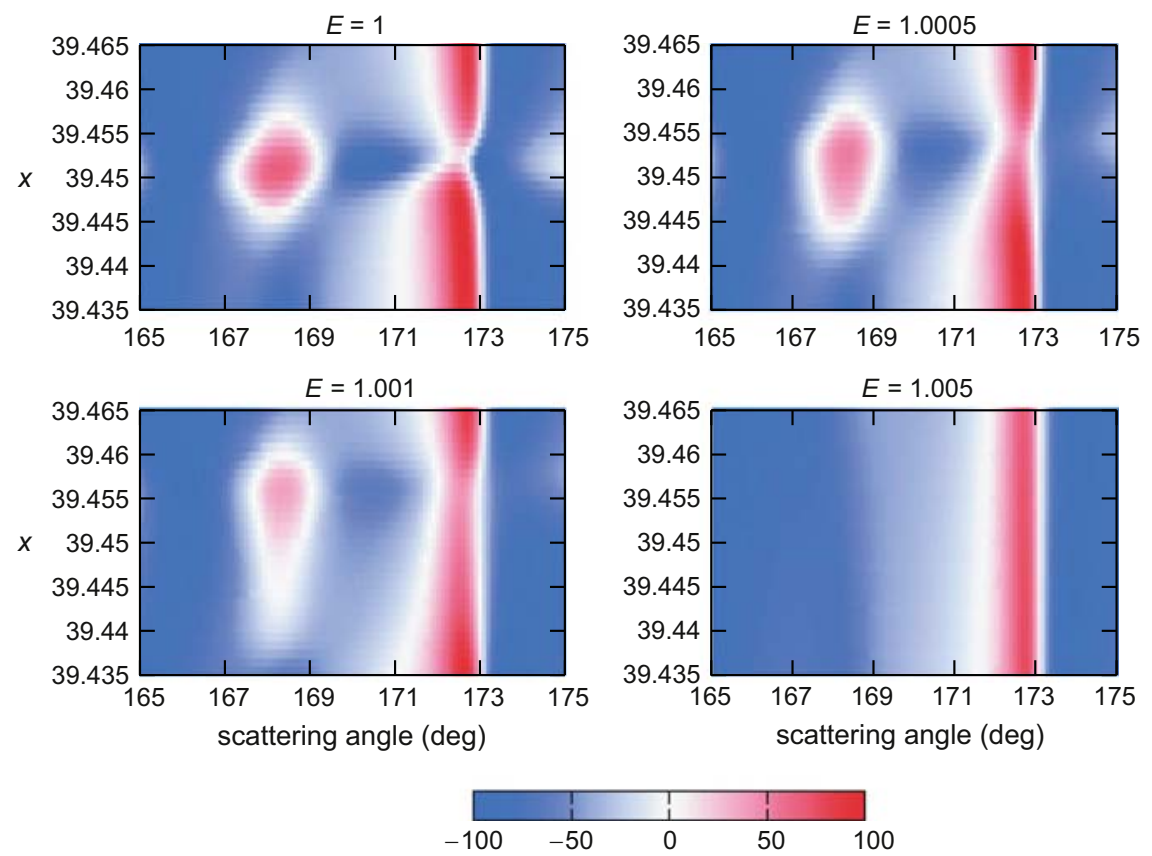

Fig. 6. High-resolution image of the degree of linear polarization $-F_{21} / F_{11}(\%)$ in the vicinity of the $x_{\text {res }}=39.4502$ resonance for a perfect sphere and volume-equivalent, randomly oriented oblate spheroids.
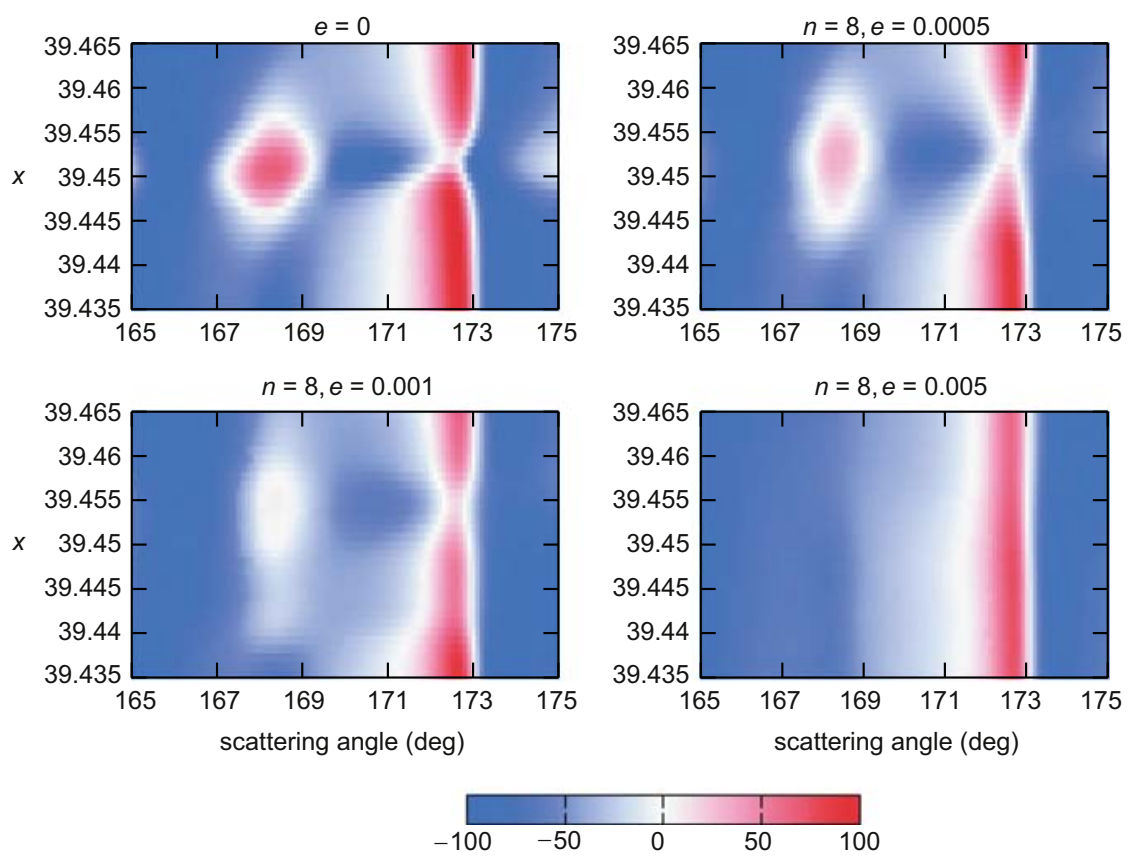

Fig. 7. As in Fig. 6, but for volume-equivalent Chebyshev particles with positive deformation parameters.

serves to level out the resonance maximum and fill in the resonance minimum, eventually resulting in the complete annihilation of the MDR.

\section{Conclusion}

The most important result of our study is that the absolute degree of asphericity (in units of size parameter) required to suppress and essentially extinguish a Lorenz-Mie MDR is approximately proportional to the resonance width (also in units of size parameter). This result implies that numerical averaging of scattering characteristics of real cloud droplets over sizes is unlikely to require the extra-fine sizeparameter resolutions that would be necessary for ideal, perfectly spherical particles. Indeed, natural ambient disturbances can be expected to induce a degree of asphericity of 
real cloud droplets and thereby annihilate MDRs with widths smaller than a certain threshold value $\tilde{W}_{x}$. Unfortunately, the absence of requisite experimental data makes this threshold value currently unknown.

Precise T-matrix computations for nearly-spherical particles remain excessively time-consuming and will not replace the operational use of the Lorenz-Mie theory in the foreseeable future. Therefore, in view of our results, one will eventually need to develop an automatic computational Lorenz-Mie procedure eliminating the contribution of the "artificial" MDRs with widths $W_{x}<\tilde{W}_{x}$. One can think of at least two alternative ways of addressing this problem. For example, if the nonsphericity of natural cloud droplets is likely to extinguish only super-narrow MDRs then it is possible that the use of a relatively large $\Delta x$ in the numerical integration over particle sizes will make the probability of an integration quadrature points hitting a resonance negligibly small. If so, the direct use of the Lorenz-Mie scattering code can be expected to generate results sufficiently accurate for most practical applications. Alternatively, one can use a recursive procedure examining the Lorenz-Mie results for triplets of sequential quadrature points in order to see if the result for the middle point deviates significantly from those for the bracketing points. If so, the artificial Lorenz-Mie resonance can be eliminated by using a simple interpolation of the bracketing results. It would be worthwhile to examine thoroughly both approaches and develop an efficient computer program suitable for use in operational calculations.

\section{Acknowledgments}

We thank S. Hill for providing his computer program for the calculation of Lorenz-Mie MDR locations and widths. This research was partly funded by the NASA Radiation Sciences Program managed by Hal Maring and by the NASA Remote Sensing Theory Program managed by Lucia Tsaoussi. We also acknowledge support from the National Academy of Sciences of Ukraine under the Main
Astronomical Observatory GRAPE/GPU/GRID Computing Cluster Project.

\section{References}

[1] Nussenzveig HM. Light tunneling in clouds. Appl Opt 2003;42: 1588-93.

[2] Chýlek P. Partial-wave resonances and the ripple structure in the Mie normalized extinction cross section. J Opt Soc Am 1976;66: 285-7.

[3] Hill SC, Benner RE. Morphology-dependent resonances. In: Barber PW, Chang RK, editors. Optical effects associated with small particles. Singapore: World Scientific; 1988. p. 3-61.

[4] Mishchenko MI, Travis LD, Lacis AA. Scattering, absorption, and emission of light by small particles. Cambridge: Cambridge University Press; 2002.

[5] Zender CS, Talamantes J. Solar absorption by Mie resonances in cloud droplets. J Quant Spectrocs Radiat Transf 2006;98:122-9.

[6] Mishchenko MI, Lacis AA. Morphology-dependent resonances of nearly spherical particles in random orientation. Appl Opt 2003;42: 5551-6.

[7] Pruppacher HR, Klett JD. Microphysics of clouds and precipitation. Dordrecht: Springer; 2010.

[8] Hale GM, Querry MR. Optical constants of water in the 200-nm to 200- $\mu \mathrm{m}$ wavelength region. Appl Opt 1973;12:555-63.

[9] van de Hulst HC. Light scattering by small particles. New York: Wiley; 1957.

[10] Chýlek P, Zhan J. Interference structure of the Mie extinction cross section. J Opt Soc Am A 1989;6:1846-51.

[11] Mishchenko MI, Travis LD. Capabilities and limitations of a current FORTRAN implementation of the $T$-matrix method for randomly oriented rotationally symmetric scatters. J Quant Spectrocs Radiat Transf 1998;60:309-24.

[12] Waterman PC. Symmetry, unitarity, and geometry in electromagnetic scattering. Phys Rev D 1971;3:825-39.

[13] Wiscombe WJ, Mugnai A. Single scattering from nonspherical Chebyshev particles: a compendium of calculations (NASA ref. publ. NASA RP-1157). Washington, DC: National Aeronautics and Space Administration; 1986.

[14] Chýlek P, Kiehl JT, Ko MKW. Narrow resonance structure in the Mie scattering characteristics. Appl Opt 1978;17:3019-21.

[15] Hill SC, Rushforth CK, Benner RE, Conwell PR. Sizing dielectric spheres and cylinders by aligning measured and computed resonance locations: algorithm for multiple orders. Appl Opt 1985;24: 2380-90.

[16] Chýlek P. Large-sphere limits of the Mie-scattering functions. J Opt Soc Am 1973;63:699-706.

[17] Probert-Jones JR. Resonance component of backscattering by large dielectric spheres. J Opt Soc Am A 1984;1:822-30. 